\title{
New bounds for Morse clusters
}

\author{
Tamás Vinkó • Arnold Neumaier
}

Received: 23 June 2005 / Accepted: 13 February 2007 / Published online: 13 April 2007

(C) Springer Science+Business Media B.V. 2007

\begin{abstract}
This paper presents new, simple arguments improving the lower bounds for the total energy and the minimal inter-particle distance in minimal energy atom cluster problems with interactions given by a Morse potential, where the atom separation problem is difficult due to the finite energy at zero atom separation. Apart from being sharper than previously known bounds, they also apply for a wider range $\rho \geq 4.967$ of the parameter in the Morse potential. Most results also hold for more general pair potentials.
\end{abstract}

Keywords Atom cluster $\cdot$ Lower bounds $\cdot$ Minimal distance $\cdot$ Morse clusters

\section{Introduction}

Given a cluster of $n$ atoms in $d$-dimensional space $(d>1)$, define the coordinate vectors $x_{i} \in \mathbb{R}^{d}(i=1, \ldots, n)$ as the center of the $i$ th atom. The potential energy of the cluster $x=\left(x_{1}, \ldots, x_{n}\right) \in \mathbb{R}^{d n}$ is then defined as the sum of the two-body inter-particle pair potentials over all of the pairs, i.e.,

$$
E(x)=\sum_{i<j} v\left(r_{i j}\right)
$$

where

$$
r_{i j}=\left\|x_{i}-x_{j}\right\|_{2}
$$

T. Vinkó (凶)

Advanced Concepts Team, European Space Agency, ESTEC, Keplerlaan 1, 2201 AZ Noordwijk, The Netherlands

e-mail: Tamas.Vinko@esa.int

A. Neumaier

Fakultät für Mathematik, Universität Wien, Nordbergstrasse 15, A-1090 Wien, Austria

e-mail: Arnold.Neumaier@univie.ac.at 
is the Euclidean distance of $x_{i}$ and $x_{j}$ and $v(r)$ is the value of the pair potential for two particles at distance $r$. The aim of this paper is to obtain lower bounds for the total energy and for the minimal interatomic distance in the structure with minimal total energy (1) in case that the pair potential is a Morse potential,

$$
v_{\rho}(r)=e^{\rho(1-r)}\left(e^{\rho(1-r)}-2\right),
$$

where $\rho>\ln 2$ is a parameter. Most results hold more generally for pair potentials $v(r)$ which are a continuous, piecewise differentiable function strictly decreasing for $r \leq s$ and increasing for $r \geq s$, with global minimum value $v(s)<0$, positive for small $r$, and approaching zero from below for $r \rightarrow \infty$. $v$ has a unique zero, which is denoted by $t$. Clearly, $t<s$ and

$$
v(t)=0, \quad v(r)<0 \quad \text { for } r>t .
$$

For the Morse potential, $s=1, v(s)=-1$, and

$$
t=1-\frac{\ln 2}{\rho}
$$

The existence of a positive zero requires $\rho>\ln 2$.

\subsection{Previous results}

The book by Ruelle [8] contains in Sect. 2.3 (see also the references given there) investigations for general pair potentials. Ruelle calls a pair potential function stable if the associated total energy of a cluster is bounded from below by a linear function of the cluster size, and proves sufficient conditions for stability (see Propositions 3.2.7 and 3.2.8 in Ref. [8]) but without giving explicit formulas for the resulting bounds. Ruelle's results apply to the Morse potential for $\rho>\ln 16(\approx 2.7726)$, giving a linear lower bound on the energy but nothing about atom separation.

Most other rigorous work was done for the Lennard-Jones interaction; see, e.g., $[1,5,9,12,13]$. For Morse clusters, the atom separation problem is significantly more difficult due to the finite energy at zero atom separation. Indeed, the first size-independent lower bounds for the interatomic distance in the optimal structures were obtained only in 2002 by Locatelli and Schoen [4], using complicated geometric arguments establishing such a bound for $d=3$ and $\rho \geq 6$. Then Vinkó [10], obtained - for general potential functions satisfying natural asymptotic properties-size-independent lower bounds on the minimal distance and linear lower bounds on the total energy which improved the results of [4], and Schachinger et al. [9] improved these further. All these results work only for $\rho \geq 6$, since they are based upon crucial estimates of [4].

The method presented in this paper improves these lower bounds further, using arguments much simpler than those of [4]. Moreover, we find size-independent lower bounds on the minimal distance already for $\rho \geq 4$.967. Most arguments apply to more general pair potentials. All numerical computations were done both with MuPAD [6] and Mathematica [11], to be sure of the correctness of the numerical results we obtained. Note that Mathematica provided faster evaluation times than MuPad. 


\subsection{Notation}

The following notation will be used. A global minimizer of the function $E$ is any configuration $x^{*} \in \mathbb{R}^{d n}$ with

$$
E^{*}:=E\left(x^{*}\right)=\min _{x \in \mathbb{R}^{d n}} E(x)
$$

where $d>1$ is the dimension of the space containing the cluster. (Of interest are mainly $d=2$ and $d=3$.) Let $r_{i j}$ be the Euclidean distance of the points $x_{i}^{*}$ and $x_{j}^{*}(i, j=1, \ldots, n)$. The potential energy of particle $i$ in an arbitrary configuration $x$ is defined as

$$
E_{i}(x)=\sum_{i \neq j} v\left(\left\|x_{i}-x_{j}\right\|\right), \quad i=1, \ldots, n
$$

and we put

$$
E_{i}^{*}=E_{i}\left(x^{*}\right)
$$

Clearly, the total energy is

$$
E(x)=\frac{1}{2} \sum_{i=1}^{n} E_{i}(x) .
$$

If the number of atoms is to be emphasized, the notation $E^{*}(n)$ and $E_{i}^{*}(n)$ is used for the optimal total energy and for the optimal potential energy of particle $i$, respectively.

We write $R_{k}$ for the minimum over $i$ of the $k$ th smallest distance of some atom from $x_{i}$. Then $R_{1}=0$, and

$$
R_{2}=r_{\min }:=\min _{i, j} r_{i j}, \quad i, j=1, \ldots, n
$$

is the minimal distance in the optimal configuration. The $R_{k}$ form a not necessarily increasing sequence. We give some atom (to be determined later) the label 1 and label the remaining atoms such that $r_{i}:=r_{1 i}$ satisfies

$$
0=r_{1} \leq r_{2} \leq \cdots \leq r_{n}
$$

Then (5) implies $r_{i} \geq R_{i}$ for $i=2, \ldots, n$.

\section{Energy bounds}

In this section, we prove bounds on the optimal total energy. We first generalize considerations by Maranas and Floudas [5] for the Lennard-Jones potential.

Lemma 1 An optimal n-atom cluster has total energy bounded by

$$
-\frac{n(n-1)}{2}|v(s)| \leq E^{*}(n) \leq-d(n-d+1)|v(s)| .
$$


Proof Since $v\left(r_{i j}\right)-v(s) \geq 0$, we have

$$
\begin{aligned}
E^{*}(n) & =\sum_{i<j}\left(v\left(r_{i j}\right)-v(s)+v(s)\right) \\
& =\sum_{i<j}\left(v\left(r_{i j}\right)-v(s)\right)+\sum_{i<j} v(s) \\
& \geq-\frac{n(n-1)}{2}|v(s)|
\end{aligned}
$$

giving the lower bound.

If we construct a cluster containing $n$ atoms where $n-d$ particles are in a position that each of them touches (i.e., has minimal distance $s$ to) $d$ others, starting with $d$ particles in such a way that the distances between these points are $s$ (i.e., a line segment in dimension 2, an equilateral triangle in dimension 3, and so on), we get a cluster of total energy $-d|v(s)|-d(n-d)|v(s)|+M \leq-d(n-d+1)|v(s)|$ with nonpositive $M$, which is the sum of the pair potential values $v(r)$ in case of $r$ is greater than $s$. Thus, this is an upper bound for the total energy of the optimal structure. Since $M<0$, the upper bound follows.

In the following, we shall assume that, in the optimal configuration, the potential energy of particle $i$ is bounded by

$$
-(n-1)|v(s)| \leq E_{i}^{*}(n)<-e_{d}|v(s)|
$$

for some constant $e_{d}>0$ independent of the dimension and the size of the given optimal cluster. It is likely that (7) holds for $n>d=3$ with $e_{d}=d$ since in the optimal structure, every atom has most likely at least $d$ contacts. But showing this rigorously seems to be nontrivial, and we only establish

Lemma 2 (7) holds with $e_{d}=1$.

Proof To prove the upper bound, let $k=n$ if $i \neq n$ and $k=n-1$ if $i=n$, and define the configuration $z=\left(z_{1}, \ldots, z_{n}\right)$ in such a way that $z_{j}=x_{j}^{*}$ for all $j \neq i,\left\|z_{i}-z_{k}\right\|=s$ and $\left\|z_{i}-z_{l}\right\| \geq s$ for all $l \neq i$. Then place the atom $z_{i}$ on the line determined by the origin and the coordinates of $z_{k}$ in such a way that $z_{i}$ has the maximal $r_{j}$ value. Thus, $E_{i}(z)<-|v(s)|$. By construction of $z$,

$$
E^{*}-E_{i}^{*}=E(z)-E_{i}(z) .
$$

Since $E_{i}(z)<-|v(s)|$ and

$$
E^{*}-E_{i}^{*}=E(z)-E_{i}(z)>E(z)+|v(s)| \geq E^{*}+|v(s)|
$$

we find the upper bound $E_{i}^{*}<-|v(s)|$. The lower bound comes from the fact that $v(r)$ is monotone decreasing in the interval $[0, s]$ and from the definition of $E_{i}^{*}(n)$. Indeed, the formula for $E_{i}^{*}(n)$ contains $n-1$ terms and all of them have the lower bound $-|v(s)|$.

To get size-independent lower bounds on $E_{i}^{*}$ and linear lower bounds on the total energy, we proceed to find upper and lower bounds on sums of the form

$$
\Sigma_{m}:=\sum_{k=2}^{m} v\left(r_{k}\right) .
$$


Let $N_{d}(r)$ be the maximal number of disjoint open unit balls fitting into a ball of radius $r$. By a simple volume comparison one can easily find the upper bound

$$
N_{d}(r) \leq\left\lfloor r^{d}\right\rfloor,
$$

which we shall use in the following. Any improvement in this geometric packing bound would result in corresponding improvements of our estimates depending on it.

Proposition 1 Let

$$
K(r):=\min _{m \in \mathbb{N}, R_{m}>0}(m-1) N_{d}\left(\frac{2 r}{R_{m}}+1\right) .
$$

Then $K$ is an increasing function of $r$, and

$$
k \leq K\left(r_{k}\right) \quad \text { for all } k=1,2, \ldots
$$

In particular,

$$
K(r) \leq(m-1)\left\lfloor\left(\frac{2 r}{R_{m}}+1\right)^{d}\right\rfloor \quad \text { for all } m=2,3, \ldots
$$

Proof Fix $k \geq 1$ and $m \geq 2$. We consider the set $S$ consisting of the $k$ atoms closest to atom 1 . We recursively pick an atom from $S$, starting with atom 1 , and remove it and the $m-2$ atoms nearest to it from $S$, until $S$ is empty. This picks a set of $\kappa=\lceil k /(m-1)\rceil$ atoms at mutual distance at least $R_{m}$. Thus the open balls of radius $R_{m} / 2$ around these atoms are disjoint and inside the open ball of radius $r_{k}+R_{m} / 2=\left(2 r_{k}+R_{m}\right) / 2$ around the atom labeled 1 . A scaling argument gives

$$
\kappa \leq N_{d}\left(\frac{2 r_{k}}{R_{m}}+1\right)
$$

hence

$$
k \leq(m-1) \kappa \leq(m-1) N_{d}\left(\frac{2 r_{k}}{R_{m}}+1\right) \leq(m-1)\left\lfloor\left(\frac{2 r_{k}}{R_{m}}+1\right)^{d}\right\rfloor .
$$

Proposition 2 If $r_{m} \leq s$ then

$$
\Sigma_{m} \leq-m|v(s)|+E_{1}^{*}+\int_{s}^{\infty} K(r) v^{\prime}(r) \mathrm{d} r .
$$

Moreover, if $m \geq 2$ and $R_{m} \leq s$ then

$$
(m-1) v\left(R_{m}\right)+\left(m+e_{d}\right)|v(s)| \leq \int_{s}^{\infty} K(r) v^{\prime}(r) \mathrm{d} r .
$$

Proof Let first $m$ be the largest integer with $r_{m} \leq s$. Then

$$
K(r) \geq K\left(r_{m}\right) \geq m \text { for } r \geq s
$$


by Proposition 1 , and $r_{m+1}>s$, hence $v\left(r_{k+1}\right)-v\left(r_{k}\right) \geq 0$ for $k \geq m+1$. Therefore, with $r_{n+1}=\infty, v(\infty)=0$, we have

$$
\begin{aligned}
\sum_{k=m+1}^{n} k\left(v\left(r_{k+1}\right)-v\left(r_{k}\right)\right) & \leq \sum_{k=m+1}^{n} K\left(r_{k}\right) \int_{r_{k}}^{r_{k+1}} v^{\prime}(r) \mathrm{d} r \\
& \leq \sum_{k=m+1}^{n} \int_{r_{k}}^{r_{k+1}} K(r) v^{\prime}(r) \mathrm{d} r=\int_{r_{m+1}}^{\infty} K(r) v^{\prime}(r) \mathrm{d} r .
\end{aligned}
$$

The left-hand-side equals

$$
-m v\left(r_{m+1}\right)-\sum_{k=m+1}^{n} v\left(r_{k}\right)=-m v\left(r_{m+1}\right)-E_{1}^{*}+\Sigma_{m}
$$

and since $\int_{r}^{\infty} v^{\prime}(r) \mathrm{d} r=-v(r)$, we find

$$
\begin{aligned}
\Sigma_{m} & \leq E_{1}^{*}+\int_{r_{m+1}}^{\infty}(K(r)-m) v^{\prime}(r) \mathrm{d} r \leq E_{1}^{*}+\int_{s}^{\infty}(K(r)-m) v^{\prime}(r) \mathrm{d} r \\
& \leq E_{1}^{*}+m v(s)+\int_{s}^{\infty} K(r) v^{\prime}(r) \mathrm{d} r .
\end{aligned}
$$

This proves (12) for the maximal allowed value of $m$. Since

$$
\Sigma_{m}-m v(s)=\sum_{k=2}^{m}\left(v\left(r_{k}\right)-v(s)\right)-v(s)
$$

is a sum of non-negative numbers, the left-hand-side is monotone increasing in $m$; thus (12) also holds for all smaller values of $m$.

By definition of $R_{m}$, one can label some atom as 1 such that $r_{m}=R_{m}$. In this case, we have for $k<m$ the trivial lower bound

$$
\Sigma_{m} \geq(m-1) v\left(R_{m}\right) .
$$

Combining this inequality with (12) and with $E_{1}^{*}<-e_{d}|v(s)|$ gives (13).

The above argument can be improved slightly with the following considerations. For integers $m \geq 1$ and real numbers $r^{\prime}, r$, let $K_{m}\left(r^{\prime}, r\right)$ be the number of $k>m$ such that $\max \left(t, r^{\prime}\right) \leq r_{k} \leq r$. Clearly, $K_{m}\left(r^{\prime}, r\right)$ is a decreasing function of $m$ and

$$
K_{m}\left(r^{\prime}, r\right) \leq K(r)-m \text { for all } r \geq r_{m} .
$$

A bound on the value $K_{m}\left(r^{\prime}, r\right)$ can be found as follows: Consider each $r_{k}$ as a center of an open ball with radius $r_{\min } / 2$. The number of such balls that can be packed into the big ball with radius $r+r_{\min } / 2$ cannot exceed $\left(2 r / r_{\min }+1\right)^{d}$. On the other hand, since $r^{\prime} \leq r_{k}$, we can drop out the small balls from the ball with radius $r^{\prime}-r_{\min } / 2$. This estimation based on the volume of spherical shells gives

$$
K_{m}\left(r^{\prime}, r\right) \leq\left\lfloor\left(\frac{2 r}{r_{\min }}+1\right)^{d}-\max \left(m, \frac{2 r^{\prime}}{r_{\min }}-1\right)^{d}\right\rfloor .
$$

In the following, we shall always define $r^{\prime}$ in terms of $r \geq s$ by

$$
v\left(r^{\prime}\right)=v(r), \quad r^{\prime} \leq s \leq r .
$$

(Closed formulas for $r^{\prime}$ exist for the specific pair potentials in the application part.) 
Proposition 3 The sum (8) is bounded by $\Sigma_{m} \leq E_{1}^{*}+\int_{s}^{\infty} K_{m}\left(r^{\prime}, r\right) v^{\prime}(r) \mathrm{d} r$.

Proof Indeed,

$$
\begin{aligned}
\Sigma_{m}-E_{1}^{*} & =-\sum_{k>m} v\left(r_{k}\right) \leq \sum_{k>m, r_{k} \geq t} \int_{0}^{-v\left(r_{k}\right)} \mathrm{d} \rho \\
& =\int_{0}^{-v\left(r_{k}\right)}\left|\left\{k>m \mid r_{k} \geq t ;-v\left(r_{k}\right) \geq \rho\right\}\right| \mathrm{d} \rho \\
& =\int_{s}^{\infty} K_{m}\left(r^{\prime}, r\right) v^{\prime}(r) \mathrm{d} r,
\end{aligned}
$$

where we made the substitution $\rho=-v(r)$ with $r \geq s$.

Note that this estimate combined with (14) for $r_{m} \leq s$ implies Proposition 2. However, combining it with (15) gives stronger bounds.

\section{Theorem 1 If}

$$
B:=\int_{s}^{\infty} K_{1}\left(r^{\prime}, r\right) v^{\prime}(r) d r<\infty
$$

then

$$
E_{i}^{*} \geq-B \text { for all } i=1, \ldots, n .
$$

Moreover, for any constant B satisfying (19),

$$
-\frac{B}{2} n \leq E^{*} \text {. }
$$

Proof The special case $m=1$ of (17) gives

$$
0=\Sigma_{1} \leq E_{1}^{*}+\int_{s}^{\infty} K_{1}\left(r^{\prime}, r\right) v^{\prime}(r) \mathrm{d} r=E_{1}^{*}+B,
$$

which leads to (19) for $i=1$. Since the choice of the label 1 is arbitrary, (19) holds for all $i$. Finally, (20) follows from (4).

Corollary 1 If $q$ is a lower bound on the minimal inter-particle distance $r_{\min }$ then (19) holds with

$$
B:=\int_{s}^{\infty}\left\lfloor\left(\frac{2 r}{q}+1\right)^{d}-\max \left(2, \frac{2 r^{\prime}}{q}-1\right)^{d}\right\rfloor v^{\prime}(r) \mathrm{d} r .
$$

Proof Using (15) for $m=2$, we can bound $B$ as defined in (18) by

$$
\begin{aligned}
B & \leq \int_{s}^{\infty}\left\lfloor\left(\frac{2 r}{r_{\min }}+1\right)^{d}-\max \left(2, \frac{2 r^{\prime}}{r_{\min }}-1\right)^{d}\right\rfloor v^{\prime}(r) \mathrm{d} r \\
& \leq \int_{s}^{\infty}\left\lfloor\left(\frac{2 r}{q}+1\right)^{d}-\max \left(2, \frac{2 r^{\prime}}{q}-1\right)^{d}\right\rfloor v^{\prime}(r) \mathrm{d} r .
\end{aligned}
$$


As mentioned in the introduction, Ruelle [8] calls a potential function stable if the energy of the optimal cluster is bounded below by a multiple of the cluster size. We summarize his sufficient conditions for stability in Ref. [8, Sect. 3.2.6]. For Morse clusters, it gives a lower bound for the energy proportional to $n$ under weaker conditions than other known arguments.

Proposition 4 (Ruelle [8]) If the pair potential $v$ is of positive type and $v(0)$ is finite then it is stable, and

$$
-\frac{n}{2} v(0) \leq E^{*}
$$

Here a continuous function $f$ is of positive type if, for arbitrary $x_{1}, \ldots, x_{n}$,

$$
\sum_{i=1}^{n} \sum_{j=1}^{n} f\left(x_{i}-x_{j}\right) \geq 0
$$

Proof For the optimal configuration $x^{\star}$,

$$
0 \leq \sum_{i=1}^{n} \sum_{j=1}^{n} v\left(\left\|x_{i}^{*}-x_{j}^{*}\right\|\right)=n v(0)+2 \sum_{i<j} v\left(\left\|x_{i}^{*}-x_{j}^{*}\right\|\right)
$$

hence

$$
-\frac{v(0)}{2} n \leq \sum_{i<j} v\left(\left\|x_{i}^{*}-x_{j}^{*}\right\|\right)
$$

In general it is not trivial to show that a pair potential function is of positive type, but Ruelle [8] quotes the known result [2] that $f$ is of positive type if and only if the Fourier transform of $f$ is of positive type. More generally, it clearly suffices for the desired conclusion that $f$ is bounded below by a function whose Fourier transform of $f$ is of positive type.

\section{Bounds on the minimal distance}

Corollary 1 depends on a lower bound for the minimal inter-particle distance. This section is devoted to obtain such lower bounds. Note that by Lemma 2 the following certainly holds with $e_{d}=1$.

Lemma 3 If $n>2+e_{d}$ then

$$
q(n)=w\left(\left(n-2-e_{d}\right)|v(s)|\right)
$$

is a lower bound for the minimal inter-particle distance in the optimal configuration. Here $w$, defined by

$$
w(x)= \begin{cases}r, & \text { iff } x=v(r) \quad \text { and } r \leq s, \\ 0, & \text { otherwise }\end{cases}
$$

is the unique solution of $v(w(x))=\min (x, v(0))$.

型 Springer 
Proof Let $E_{1}^{*}(n)$ be the term which contains the minimal distance in the optimal structure. Using (7), we find

$$
\begin{aligned}
-e_{d}|v(s)| & >\sum_{j=2}^{n} v\left(r_{j}\right) \\
& =\sum_{j=3}^{n} v\left(r_{j}\right)+v\left(r_{2}\right) \\
& \geq-(n-2)|v(s)|+v\left(r_{2}\right) .
\end{aligned}
$$

Rearranging the inequalities one obtains $v\left(r_{2}\right)<\left(n-2-e_{d}\right)|v(s)|$, which implies the bound.

Lemma 4 In the optimal configuration the minimal interatomic distance is always less than or equal to the minimizer point of the pair potential function, i.e., $r_{\min } \leq$ s holds.

Proof Suppose that in the optimal configuration $r_{\min }>s$. We know that function $v$ is increasing for $r \geq s$. Hence, rescaling all of the distances such that $r_{\min }=s$ decreases the total energy. Thus $r_{\min } \leq s$.

Theorem 2 Let $[\underline{R}, \bar{R}] \subseteq[0, s]$ be such that

$$
\begin{gathered}
\int_{s}^{\infty}\left\lfloor\left(\frac{2 r}{R}+1\right)^{d}\right\rfloor v^{\prime}(r) \mathrm{d} r \leq v(R)+|v(s)| \quad \text { for all } R \in[\underline{R}, \bar{R}], \\
\int_{s}^{\infty}\left\lfloor\left(\frac{2 r}{\bar{R}}+1\right)^{d}\right\rfloor v^{\prime}(r) \mathrm{d} r<\min \left\{v(\underline{R})+|v(s)|, \frac{1}{2} v(\underline{R})+\left(1+\frac{e_{d}}{2}\right)|v(s)|\right\} .
\end{gathered}
$$

Then the function defined by

$$
f(q):=v(q)+\left(2+e_{d}\right)|v(s)|-\int_{s}^{\infty}\left\lfloor\left(\frac{2 r}{q}+1\right)^{d}\right\rfloor v^{\prime}(r) \mathrm{d} r
$$

has a smallest zero $q$ in $] \bar{R}, \infty\left[\right.$, and we have $r_{\min } \geq q$.

Proof For any integer $m \geq 2$ we find from (11) and (13) that $R=R_{m}$ satisfies

$$
(m-1) v(R)+\left(m+e_{d}\right)|v(s)| \leq \int_{s}^{\infty}(m-1)\left\lfloor\left(\frac{2 r}{R}+1\right)^{d}\right\rfloor v^{\prime}(r) \mathrm{d} r
$$

hence

$$
v(R)+|v(s)|<v(R)+\frac{m+e_{d}}{m-1}|v(s)| \leq \int_{s}^{\infty}\left\lfloor\left(\frac{2 r}{R}+1\right)^{d}\right\rfloor v^{\prime}(r) \mathrm{d} r .
$$

This contradicts (26) unless

$$
R_{m}<\underline{R} \text { or } R_{m}>\bar{R} \text {. }
$$

If the first case can happen for some $m \geq 2$, let $m$ be the largest integer such that $R_{m}<\underline{R}$. Then $R_{m+1}>\bar{R}$, hence

$$
K(r) \leq m\left\lfloor\left(\frac{2 r}{R_{m+1}}+1\right)^{d}\right\rfloor \leq m\left\lfloor\left(\frac{2 r}{\bar{R}}+1\right)^{d}\right\rfloor
$$


and since $v(\underline{R}) \leq v\left(R_{m}\right)$, we find from (13) that

$$
\frac{1}{m}\left((m-1) v(\underline{R})+\left(m+e_{d}\right)|v(s)|\right) \leq \int_{s}^{\infty}\left\lfloor\left(\frac{2 r}{\bar{R}}+1\right)^{d}\right\rfloor v^{\prime}(r) \mathrm{d} r .
$$

The left-hand-side is monotone in $m$, hence extremal at the boundary, and since $m \geq 2$, this contradicts (27). Thus the first case cannot happen. In particular, we find for $m=2$ that

$$
r_{\min }=R_{2}>\bar{R}
$$

Since (13) implies for $m=2$ that $f\left(r_{\min }\right) \leq 0$ and (26) implies $f(\bar{R})>2|v(s)|>0$, the intermediate value theorem implies that $f$ has a zero in $] \bar{R}, \infty\left[\right.$, and that $r_{\text {min }}$ cannot be smaller than the smallest such zero.

Corollary 2 If there is some $R \in[0, s]$ such that

$$
\int_{s}^{\infty}\left\lfloor\left(\frac{2 r}{R}+1\right)^{d}\right\rfloor v^{\prime}(r) \mathrm{d} r<\min \left\{v(R)+|v(s)|, \frac{1}{2} v(R)+\frac{3}{2}|v(s)|\right\}
$$

then the assumptions of the theorem are satisfiable, and there is a positive $n$-independent lower bound on $r_{\min }$.

Proof Take $\underline{R}=\bar{R}=R$.

Note that the assumption is automatically satisfied with $R=0$ if the potential $V(r)$ diverges for $r \rightarrow 0$, but is a nontrivial restriction for the Morse potential.

By Theorem 2, we can compute lower bound on the minimal inter-particle distance. If we take $m=2$ in formula (15) and in Proposition 3, it leads to better results. Namely, the function defined by

$$
f\left(q^{\prime}\right):=v\left(q^{\prime}\right)+e_{d}|v(s)|-\int_{s}^{\infty}\left\lfloor\left(\frac{2 r}{q}+1\right)^{d}-\max \left(2, \frac{2 r^{\prime}}{q}-1\right)^{d}\right\rfloor v^{\prime}(r) \mathrm{d} r
$$

also has a smallest zero in $] \bar{R}, \infty\left[\right.$ and then we have $r_{\min } \geq q^{\prime}$.

\section{Numerical results}

In this section, the numerical results are shown for different $\rho$ values. For $\rho=6$, the Morse and the scaled Lennard-Jones pair potential are related; they have the same curvature at the minimum point $r=1$. In the context of global optimization, the cases $\rho>6$ are most interesting, since these are more difficult problems than finding the optimal Lennard-Jones structure [3]. On the other hand, finding minimal interatomic distance in the optimal Morse cluster becomes more difficult as $\rho$ becomes smaller and the pair potential becomes less repulsive at small distances.

4.1 Size dependent bound for the minimal distance

Lemma 3 gives

$$
\left(e^{\rho(1-r)}-1\right)^{2}-1 \leq\left(n-2-e_{d}\right)\left|v_{\rho}(s)\right|
$$


Table 1 Lower bounds on minimum inter-particle distance and total energy of optimal Morse clusters

\begin{tabular}{lllllllr}
\hline$\rho$ & $t$ & $\underline{R}$ & $\bar{R}$ & $\begin{array}{l}q \text { from } \\
\text { formula }(29)\end{array}$ & $\begin{array}{l}q \text { from } \\
\text { L\&S [4] }\end{array}$ & $\begin{array}{l}q \text { from } \\
\text { Sch.[9] }\end{array}$ & $\begin{array}{l}\text { New bounds } \\
\text { for } E_{\rho}^{*}\end{array}$ \\
\hline 15 & 0.95379 & 0.00001 & 0.86424 & 0.865691 & 0.715166 & 0.865230 & $-20.6118 n$ \\
14 & 0.95049 & 0.00197 & 0.85320 & 0.854724 & 0.694918 & 0.854303 & $-21.5684 n$ \\
13 & 0.94668 & 0.00039 & 0.84018 & 0.841826 & 0.671606 & 0.841431 & $-22.7337 n$ \\
12 & 0.94224 & 0.00077 & 0.82460 & 0.826440 & 0.644492 & 0.826034 & $-24.1842 n$ \\
11 & 0.93699 & 0.00152 & 0.80559 & 0.807692 & 0.612565 & 0.807275 & $-26.0923 n$ \\
10 & 0.93068 & 0.00302 & 0.78187 & 0.784318 & 0.574381 & 0.783890 & $-28.7126 n$ \\
9 & 0.92298 & 0.00608 & 0.75135 & 0.754293 & 0.527627 & 0.753859 & $-32.5332 n$ \\
8 & 0.91336 & 0.01250 & 0.71045 & 0.714143 & 0.467709 & 0.713711 & $-38.6074 n$ \\
7 & 0.90097 & 0.02663 & 0.65212 & 0.657166 & 0.375988 & 0.656752 & $-49.7140 n$ \\
6 & 0.88448 & 0.06167 & 0.55928 & 0.567463 & 0.113522 & 0.567097 & $-76.3745 n$ \\
5 & 0.86137 & 0.20982 & 0.33235 & 0.371212 & - & - & $-245.8110 n$ \\
4.967 & 0.86045 & 0.23439 & 0.30471 & 0.356997 & - & - & $-272.8310 n$ \\
\hline
\end{tabular}

Since $s=1$, we have $v_{\rho}(s)=-1$, and we conclude that

$$
q(n)=\max \left\{0,1-\rho^{-1} \ln \left(1+\sqrt{n-2-e_{d}}\right)\right\}
$$

is a lower bound for the minimal inter-particle distance of an optimal Morse cluster with $n>2+e_{d}$ particles. This formula yields a strictly positive bound if

$$
n \leq\left\lfloor\left(2+e_{d}\right)+\frac{e^{\rho}\left(e^{\rho}-2\right)}{\left|v_{\rho}(s)\right|}\right\rfloor \text {. }
$$

4.2 Size independent bound and linear lower bound for the energy

Ruelle [7] proved that if $\rho>\ln 16 \approx 2.7726$, then the Fourier transform of the pair potential $v_{\rho}$ is of positive type, hence it is stable by Bochner's theorem [2] and Proposition 4. The resulting linear lower bound,

$$
-\frac{v_{\rho}(0)}{2} n \leq M_{\rho}^{*}, \quad \rho>\ln 16
$$

is quite poor: For $\rho=4.967$ (the smallest value for which the condition in Corollary 2 holds) and for $\rho=15$ formula (31) gives $-1.0166 \cdot 10^{4} n$ and $-5.3432 \cdot 10^{12} n$, respectively.

No bound on the minimal distance is available from Ruelle's argument. However, our theory applies. Table 1 contains the results of the application of formula (29) for Morse clusters, together with the previous results from Locatelli and Schoen [4] and Schachinger et al. [9]. Those of Vinkó [10] are intermediate in quality, and are not reported for space reasons. The last line $(\rho=4$.967) shows the smallest $\rho$ where formula (29) could be applied according to Theorem 2. The linear lower bounds for $E_{\rho}$ obtained from Theorem 1 are also presented.

\section{Conclusions}

The methods were introduced in this paper are able to make lower bounds on the minimal interatomic distance and on the total energy in optimal structures of Morse 
clusters. With these methods size dependent bounds (for small configurations) and size independent bounds (for arbitrary large clusters) can be obtained. Numerical computations show that these bounds are better than the known ones for Morse clusters. Moreover, the size independent method has the advantage that is able to handle Morse clusters directly, even for small $\rho$ parameters.

Acknowledgments This work has been supported by the grants OTKA T 048377 and AÖU 60oü6.

\section{References}

1. Blanc, X.: Lower bounds for the interatomic distance in Lennard-Jones clusters. Comput. Optim. Appl. 29, 5-12 (2004)

2. Bochner, S.: Lectures on Fourier Integrals. Princeton University Press. Princeton, NJ (1959)

3. Doye, J.P.K., Leary, R.H., Locatelli, M., Schoen, F.: The global optimization of Morse clusters by potential energy transformations. INFORMS J. Comput. 16, 371-379 (2004)

4. Locatelli, M., Schoen, F.: Minimal interatomic distance in Morse-clusters. J. Glob. Optim. 22, 175$190(2002)$

5. Maranas, C., Floudas, C.: A global optimization approach for Lennard-Jones microclusters. J. Chem. Phys. 97, 7667-7678 (1992)

6. MuPAD Research Group. http://www.mupad.de

7. Ruelle, D.: Classical statistical mechanics of a system of particles. Helv. Phys. Acta 36, 183197 (1963)

8. Ruelle, D.: Statistical Mechanics - Rigorous Results. Benjamin, New York (1969)

9. Schachinger, W., Addis, B., Bomze, I.M., Schoen, F.: New results for molecular formation under pairwise potential minimization. Comput. Optim. Appl. to appear (in press)

10. Vinkó, T.: Minimal inter-particle distance in atom clusters. Acta Cybern. 17, 105-119 (2005) http://www.inf.u-szeged.hu/ tvinko/mindist_vinko.pdf

11. Wolfram Research. Mathematica. http://www.wolfram.com/products/mathematica/index.html

12. Xue, G.L., Maier, R.S., Rosen, J.B.: Minimizing the Lennard-Jones potential function on a massively parallel computer. In: Proceedings of the 6th International Conference Supercomputing, Washington, DC, USA, pp. 409-416 (1992)

13. Xue, G.L.: Minimum inter-particle distance at global minimizers of Lennard-Jones clusters. J. Glob. Optim. 11, 83-90 (1997) 